\title{
Attrition of Canadian Internet pharmacy websites: what are the implications?
}

\author{
This article was published in the following Dove Press journal: \\ Drug, Healthcare and Patient Safety \\ 14 August 2013 \\ Number of times this article has been viewed
}

\section{Michael A Veronin' \\ Kristen M Clancy ${ }^{2}$ \\ 'Rangel College of Pharmacy, Texas A\&M Health Science Center, Texas, USA; ${ }^{2}$ Christus Dubuis Health System, Texas, USA}

Background: The unavailability of Internet pharmacy websites may impact a consumer's drug purchases and health care.

Objective: To address the issue of attrition, a defined set of Canadian Internet pharmacy websites was examined at three separate time intervals.

Methods: In February to March 2006, 117 distinct, fully functional "Canadian Internet pharmacy" websites were located using the advanced search options of Google and the uniform resource locator (URL) for each website was recorded. To determine website attrition, each of the 117 websites obtained and recorded from the previous study was revisited at two later periods of time within a 4-year period.

Results: After approximately 4 years and 5 months, only 59 (50.4\%) sites were found in the original state. Thirty-four sites $(29.1 \%)$ had moved to a new URL address and were not functioning as the original Internet pharmacy. For 24 sites $(20.5 \%)$ the viewer was redirected to another Canadian Internet pharmacy site.

Conclusion: Of concern for patients if Internet pharmacy sites were suddenly inaccessible would be the disruption of continuity of care.

Keywords: Canadian Internet pharmacy, online pharmacy, website attrition, continuity of care

\section{Introduction}

The World Wide Web has been characterized as having "rapid and often uncontrolled growth" and as a consequence, website disappearance is often an unanticipated and unavoidable occurrence. ${ }^{1}$ The term "link rot" refers to a hyperlink that points to a computer or document that is moved, deleted, or no longer accessible. The link may appear functional, but when clicked on, a "not found" or similar message may appear. The unavailability or attrition of Internet websites has been described extensively in selected studies, mainly focusing on loss of references in periodicals, where the rate of uniform resource locator (URL) "decay" has been deemed a "growing threat to scientific communication." $2-4$

For example, inactive URLs have been documented in the reference sections of the highest impact scientific and medical journals: New England Journal of Medicine, Journal of the American Medical Association, and Science. It was stated, "Internet references occurred frequently and were often inaccessible within months after publication." ${ }^{4}$ The percentage of inactive Internet references increased from $3.8 \%$ at 3 months, to $10 \%$ at 15 months, and to $13 \%$ at 27 months after publication. ${ }^{4}$ The unavailability of URLs is not restricted to references in medicine or science. One study of "link rot" showed a striking rate of decline of nearly $70 \%$ after 5 years in the law literature. ${ }^{5}$
Correspondence: Michael A Veronin Social and Administrative Sciences, Department of Pharmaceutical Sciences, Rangel College of Pharmacy, Texas A\&M Health Science Center, MSC 131, 1010 West Avenue B, Kingsville, Texas 78363, USA

$\mathrm{Tel}+\mid$ 36| 22I 0755

Fax+l 36I 5934303

Email veronin@tamhsc.edu 
URL disappearance has been reported in areas other than bibliographic references as well. In a health related study, almost 59\% (108 of 184) of URL website addresses for a popular herbal remedy became inactive within 4 years. ${ }^{6}$ In another study related to academics, over $20 \%$ of several hundred hyperlinked webpages in high school science courses were monitored over a 2-year period since their creation in August, 2000. The URLs with the domain extensions “.com" and ".edu" became inactive most often."

Although much information has dealt with website attrition related to bibliographic and related references, the topic has not been examined in websites that emphasize product sales and services to consumers, such as Internet pharmacies. An Internet or online pharmacy is defined as "a website that advertises, prescribes, dispenses, and sells prescription drugs and other health and beauty products." ${ }^{8}$ Internet pharmacy websites are unique in that they exist to provide consumers with a product necessary for the continuum of health care - pharmaceuticals - in addition to related health information. ${ }^{9}$

\section{Primary objective}

The goal of this study was to examine Canadian Internet pharmacy website loss and the potential impact on health care. To accomplish this, a defined set of Internet pharmacy websites were examined at specific time intervals.

\section{Methods and procedures} Data sources

In an earlier study, a systematic survey was conducted to determine the price disparities on Canadian Internet pharmacy websites for two commonly prescribed drugs. ${ }^{10}$ Website identification and attributes were described, and a similar process was employed for this study. For Internet searches, the perspective was that of a consumer seeking to purchase these prescription medications online. In February to March 2006, 117 distinct and fully functional "Canadian Internet pharmacy" websites were located using the advanced search options of Google and the URL for each website was recorded. The keywords selected for entry into the query box of the browser included the search terms "Canadian online pharmacy," "generic simvastatin," and "generic atorvastatin" to identify sites selling these high demand drug products. A website was judged to be relevant and was selected, if within the site, indications are that the site represents a Canadian Internet pharmacy whose purpose is the sale of drugs and pharmaceutical products. The Internet pharmacy website must indicate or suggest to the viewer that the operation is located in Canada, operates from Canada, or contains a familiar Canadian emblem such as a maple leaf. The original list of Canadian Internet pharmacy websites is presented in Table 1.

To determine the degree of website attrition, each of the 117 websites obtained and recorded from the previous study were revisited at two later periods of time within a 4-year period. In July 2009 and July 2010, the previously recorded URL for each website was entered into the address field of the browser, Internet Explorer 7. It was documented whether the original website could not be found, had moved to a different URL location, or if the URL and site location was found unchanged from the original search. Websites were assessed for accessibility, functionality, and whether or not the website claimed to sell medications that were not, in fact, from a Canadian source.

Since it is conceivable that inaccessibility of websites may be due to temporary server problems, another attempt was made to access the sites at different periods of time. For each "HTTP Error 404," "Server Not Found," or similar message obtained from the initial URL checks, an attempt to access these sites was made during the determined time periods on various days and times of day in the manner described above.

\section{Main outcome and results}

In July 2009, after a period of approximately 3 years and 5 months, when each URL address from the original set of 117 websites was re-entered into the address field of the browser, 53 (45.3\%) sites could not be found in the original state.

Of the 53 sites, 21 website addresses (17.9\%) led to "dead ends" which meant that the website was inaccessible on three separate occasions, there was no current hosting provided to the domain name, or the viewer was informed that the site was no longer functioning. Twelve websites $(10.3 \%)$ were no longer functioning as online websites, even though the domain may be currently owned. When accessing this page, the viewer is exposed to listings similar to what one would find at another search engine which may include links to other online pharmacies. Twenty $(17.1 \%)$ had moved to a new URL address (ie, no longer functioning as the original online pharmacy) and automatically redirected the visitor to another Canadian Internet pharmacy website. Sixty-four (54.7\%) sites were found to be active from the original URLs identified previously.

In July 2010, approximately a year after the sites were revisited the first time (a total period of approximately 4 years 
Table I Canadian Internet pharmacies: status at three different time periods

\begin{tabular}{|c|c|c|c|}
\hline Website (name) & February 2006 & July 2009 & July 2010 \\
\hline I. http://canadamednet.com (www.canadamednet.com) & Original site active & Original site active & Original site active \\
\hline \multirow[t]{2}{*}{ 2. http://canadapharmacydirect.com (Canada Pharmacy direct) } & Original site active & Redirect to & Redirect to \\
\hline & & CanadaDrugs.com & CanadaDrugs.com \\
\hline 3. http://www.canada2u.net (Canada2u.net) & Original site active & Original site active & Original site active \\
\hline $\begin{array}{l}\text { 4. http://drugstogo.net/canadian-online-pharmacy.html } \\
\text { (DrugsToGo.Net) }\end{array}$ & Original site active & Domain expired; for sale & URL not found \\
\hline 5. http://www.77canadapharmacy.com (77Canada Pharmacy.com) & Original site active & Original site active & Original site active \\
\hline 6. http://www.abconlinepharmacy.com (abc online pharmacy.com) & Original site active & Original site active & Original site active \\
\hline 7. http://www.affordmeds.com (AffordMeds.com) & Original site active & Original site active & Original site active \\
\hline 8. http://www.buylowdrugs.com (Buylowdrugs) & Original site active & Original site active & Original site active \\
\hline 9. http://www.canadacheaprx.com (www.Canadacheaprx.com) & Original site active & Original site active & Original site active \\
\hline 10. http://www.canadacure.com (CanadaCure.com) & Original site active & Original site active & Original site active \\
\hline \multirow[t]{2}{*}{ II. http://www.canadadrugprices.com (CanadaDrugPrices.com) } & Original site active & Redirect to & Redirect to \\
\hline & & $\underline{\text { Saverxcanada.com }}$ & $\underline{\text { Saverxcanada.com }}$ \\
\hline 12. *http://www.canadadrugs.com (CanadaDrugs.com) & Original site active & Original site active & Original site active \\
\hline 13. http://www.canadadrugsonline.com (CanadaDrugsOnline) & Original site active & Original site active & Original site active \\
\hline 14. http://www.canadadrugstop.com (Canadadrugstop.com) & Original site active & Original site active & Original site active \\
\hline 15. http://www.canadamedicinemart.com (Canada Medicinemart) & Original site active & $\begin{array}{l}\text { Site inactive; other } \\
\text { business }\end{array}$ & $\begin{array}{l}\text { Site inactive; other } \\
\text { business }\end{array}$ \\
\hline 16. http://www.canadamedicineshop.com (CanadaMedicineShop.com) & Original site active & Original site active & Original site active \\
\hline 17. http://www.canadamedicose.com (Canada MediCose.com) & Original site active & Original site active & Original site active \\
\hline 18. http://www.canadamednet.com (Canadamednet.com) & Original site active & Original site active & Original site active \\
\hline 19. http://www.canadameds.com (Canadameds.com) & Original site active & Original site active & Original site active \\
\hline 20. http://www.canadaonlinemeds.com (CanadaOnlineMeds.com) & Original site active & $\begin{array}{l}\text { Not active; redirect } \\
\text { to domain directory }\end{array}$ & $\begin{array}{l}\text { Not active; redirect } \\
\text { to domain directory }\end{array}$ \\
\hline 21. http://www.canadapharmacy.com (CanadaPharmacy) & Original site active & Original site active & Original site active \\
\hline 22. http://www.canadapharmusa.com (CanadaPharmUSA.com) & Original site active & $\begin{array}{l}\text { Not active; redirect } \\
\text { to domain directory }\end{array}$ & $\begin{array}{l}\text { Not active; redirect } \\
\text { to domain directory }\end{array}$ \\
\hline 23. http://www.canadarxconnection.com (CanadaRxConnection) & Original site active & Original site active & Original site active \\
\hline 24. http://www.canadarxdrugservices.com (CanadaRx Drug Services) & Original site active & $\begin{array}{l}\text { Not active; redirect } \\
\text { to domain directory }\end{array}$ & $\begin{array}{l}\text { Not active; redirect } \\
\text { to domain directory }\end{array}$ \\
\hline 25. http://www.canadarxpharmacy.com (Canada Rx Pharmacy) & Original site active & $\begin{array}{l}\text { Not active; redirect } \\
\text { to domain directory }\end{array}$ & $\begin{array}{l}\text { Not active; redirect } \\
\text { to domain directory }\end{array}$ \\
\hline 26. http://www.canadian-drugs.com (Discount Canadian Drugs) & Original site active & $\begin{array}{l}\text { Redirect to } \\
\text { CanadaDrugs.com }\end{array}$ & $\begin{array}{l}\text { Redirect to } \\
\text { CanadaDrugs.com }\end{array}$ \\
\hline 27. http://www.canadiandrugsolutions.com (Canadiandrugsolutions.com) & Original site active & $\begin{array}{l}\text { Not active; redirect } \\
\text { to domain directory }\end{array}$ & $\begin{array}{l}\text { Not active; redirect } \\
\text { to domain directory }\end{array}$ \\
\hline $\begin{array}{l}\text { 28. http://www.canadianmailorderdrugstores.com } \\
\text { (Canadian Mail Order Drugstores) }\end{array}$ & Original site active & Original site active & $\begin{array}{l}\text { Not active; redirect } \\
\text { to domain directory }\end{array}$ \\
\hline 29. http://www.canadianmedservice.com (Canadian Med Service) & Original site active & Site Inactive & Site Inactive \\
\hline 30. http://www.canadian-pharmacies.com (Canadian-Pharmacies.com) & Original site active & $\begin{array}{l}\text { Redirect to cross } \\
\text { border pharmacy }\end{array}$ & $\begin{array}{l}\text { Site active as cross } \\
\text { border pharmacy }\end{array}$ \\
\hline $\begin{array}{l}\text { 31. http://www.canadianpharmacychoice.com } \\
\text { (Canadian Pharmacy Choice) }\end{array}$ & Original site active & Original site active & URL not found \\
\hline 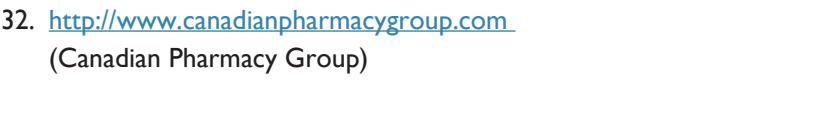 & Original site active & $\begin{array}{l}\text { Redirect to } \\
\text { CanadianPharmacy } \\
\text { Link.com }\end{array}$ & $\begin{array}{l}\text { Redirect to } \\
\text { CanadianPharmacy } \\
\text { Link.com }\end{array}$ \\
\hline $\begin{array}{l}\text { 33. http://www.canadianpharmacyinfo.com } \\
\text { (Canadianpharmacyinfo.com)' }\end{array}$ & Original site active & URL not found & URL not found \\
\hline 34. http://www.canadianpharmacylink.com (Canadian Pharmacy Link) & Original site active & Original site active & Original site active \\
\hline $\begin{array}{l}\text { 35. http://www.canadian-pharmacy-prices.com } \\
\text { (Canadian-Pharmacy-Prices.com) }\end{array}$ & Original site active & $\begin{array}{l}\text { Redirects to } \\
\text { Saverxcanada.com }\end{array}$ & $\begin{array}{l}\text { Redirects to } \\
\text { Saverxcanada.com }\end{array}$ \\
\hline 36. http://www.canadianpharmacyweb.com (CanadianPharmacyWeb) & Original site active & Original site active & Original site active \\
\hline $\begin{array}{l}\text { 37. http://www.canadianprescriptiondrugstore.com } \\
\text { (Canadian Prescription Drugstore) }\end{array}$ & Original site active & Original site active & Original site active \\
\hline $\begin{array}{l}\text { 38. http://www.canadiansupersavepharmacy.com } \\
\text { (CanadianSuperSavePharmacy.com) }\end{array}$ & Original site active & Original site active & $\begin{array}{l}\text { Redirect to } \\
\text { Candrugstore.com }\end{array}$ \\
\hline
\end{tabular}

(Continued) 
Table I (Continued)

\begin{tabular}{|c|c|c|c|}
\hline Website (name) & February 2006 & July 2009 & July 2010 \\
\hline 39. http://www.canarx.com (CanaRx) & Original site active & Original site active & Original site active \\
\hline 40. http://www.candrug.com (CanDrug) & Original site active & Original site active & Original site active \\
\hline 4I. http://www.candrugstore.com (Candrugstore.com) & Original site active & Original site active & Original site active \\
\hline 42. http://www.canpharmex.com (CanadaPharmacyExpress) & Original site active & URL not found & URL not found \\
\hline 43. http://www.cantrustrx.com (CantrustRx) & Original site active & Original site active & Original site active \\
\hline 44. http://www.CanUSAmeds.com (CanUsaMeds.com) & Original site active & Original site active & Original site active \\
\hline 45. http://www.cheaprxmeds.com (CheapRxmeds.com) & Original site active & Original site active & Original site active \\
\hline 46. http://www.christianpharmacy.com (Christian Pharmacy) & Original site active & $\begin{array}{l}\text { Not active; redirect } \\
\text { to domain directory }\end{array}$ & $\begin{array}{l}\text { Not active; redirect } \\
\text { to domain directory }\end{array}$ \\
\hline 47. http://www.crossborderpharmacy.com (Crossborderpharmacy.com) & Original site active & Original site active & Original site active \\
\hline $\begin{array}{l}\text { 48. http://www.discount-meds-canada.com } \\
\text { (Discount-Meds-Canada.com) }\end{array}$ & Original site active & $\begin{array}{l}\text { Redirects to } \\
\text { Saverxcanada.com }\end{array}$ & $\begin{array}{l}\text { Redirects to } \\
\text { Saverxcanada.com }\end{array}$ \\
\hline 49. http://www.doctorsolve.com (DoctorSolve) & Original site active & Original site active & Original site active \\
\hline 50. http://www.drugdelivery.ca (Drugdelivery.ca)* & Original site active & Original site active & $\begin{array}{l}\text { Redirect to } \\
\text { PharmacyEscrow.com }\end{array}$ \\
\hline 5I. http://www.drugsraffordable.com (Drugs R affordable.com) & Original site active & $\begin{array}{l}\text { Not active; redirect } \\
\text { to domain directory }\end{array}$ & $\begin{array}{l}\text { Not active; redirect } \\
\text { to domain directory }\end{array}$ \\
\hline 52. http://www.economedsplus.com (Economedsplus) & Original site active & $\begin{array}{l}\text { Redirect to } \\
\text { CanadaDrugs.com }\end{array}$ & $\begin{array}{l}\text { Redirect to } \\
\text { CanadaDrugs.com }\end{array}$ \\
\hline 53. http://www.expressmedscanada.com (Express Meds Canada)* & Original site active & Original site active & Original site active \\
\hline 54. http://www.federaldrugs.com (Federal Drugs) & Original site active & Original site active & Original site active \\
\hline 55. http://www.getinpharmacy.com (Getinpharmacy.com) & Original site active & URL not found & URL not found \\
\hline $\begin{array}{l}\text { 56. http://www.heartland-canada-pharmacy-connection.com } \\
\text { (Heartland Canada Pharmacy Connection) }\end{array}$ & Original site active & $\begin{array}{l}\text { Not active; redirect } \\
\text { to domain directory }\end{array}$ & $\begin{array}{l}\text { Not active; redirect } \\
\text { to domain directory }\end{array}$ \\
\hline 57. http://www.hometownmeds.com (Hometown Meds) & Original site active & $\begin{array}{l}\text { Redirect to } \\
\text { CanadaDrugs.com }\end{array}$ & $\begin{array}{l}\text { Redirect to } \\
\text { CanadaDrugs.com }\end{array}$ \\
\hline 58. http://www.icanrx.com (www.iCanRx.com) & Original site active & Original site active & Original site active \\
\hline 59. http://www.jandrugs.com (Jandrugs) & Original site active & Original site active & Original site active \\
\hline 60. http://www.lepharmacy.com (LePharmacy.com) & Original site active & $\begin{array}{l}\text { Message on site: } \\
\text { discontinued }\end{array}$ & $\begin{array}{l}\text { Message on site: } \\
\text { discontinued }\end{array}$ \\
\hline 61. http://www.libertycarerxcanada.com (Liberty Care Rx Canada) & Original site active & Original site active & Original site active \\
\hline 62. http://www.mapleleafmeds.com (Maple Leaf Meds) & Original site active & Original site active & Original site active \\
\hline 63. http://www.medcana.com (Medcana) & Original site active & $\begin{array}{l}\text { Redirect to } \\
\text { CanadaDrugs.com }\end{array}$ & $\begin{array}{l}\text { Redirect to } \\
\text { CanadaDrugs.com }\end{array}$ \\
\hline 64. http://www.medcentercanada.com (Medcenter Canada) & Original site active & Original site active & Original site active \\
\hline 65. http://www.medications4less.net (Medications 4 less) & Original site active & $\begin{array}{l}\text { Not active; "Shut down } \\
\text { by state of NJ" }\end{array}$ & $\begin{array}{l}\text { Not active; "Shut down } \\
\text { by state of NJ" }\end{array}$ \\
\hline 66. http://www.medicationscanada.com (Medicationscanada) & Original site active & Original site active & Original site active \\
\hline 67. http://www.medisave.ca (MediSave.ca) & Original site active & Original site active & Original site active \\
\hline 68. http://www.meds4u.com (Meds 4U)* & Original site active & Original site active & Original site active \\
\hline 69. http://www.medstorecanada.com (MedstoreCanada.com) & Original site active & $\begin{array}{l}\text { Not active; redirect } \\
\text { to domain directory }\end{array}$ & $\begin{array}{l}\text { Not active; redirect } \\
\text { to domain directory }\end{array}$ \\
\hline 70. http://www.mercanrx.com (MerCanRx) & Original site active & URL not found & URL not found \\
\hline 71. http://www.Nobordersrx.com (NobordersRx.com) & Original site active & $\begin{array}{l}\text { Message on site: } \\
\text { "Suspended" }\end{array}$ & $\begin{array}{l}\text { Message on site: } \\
\text { "Suspended" }\end{array}$ \\
\hline 72. http://www.norphar.com (North American Pharmacy Network) & Original site active & original site active & original site active \\
\hline 73. http://www.northcountrymeds.com (NorthCountryMeds.com) & Original site active & $\begin{array}{l}\text { Redirect to } \\
\text { CanadaDrugs.com }\end{array}$ & $\begin{array}{l}\text { Redirect to } \\
\text { CanadaDrugs.com }\end{array}$ \\
\hline 74. http://www.northcountryrx.com (North Country Rx) & Original site active & URL not found & URL not found \\
\hline 75. http://www.oneworldrx.com (OneWorldRx) & Original site active & $\begin{array}{l}\text { Redirect to } \\
\text { Prescriptnet.com }\end{array}$ & $\begin{array}{l}\text { Redirect to } \\
\text { Prescriptnet.com }\end{array}$ \\
\hline 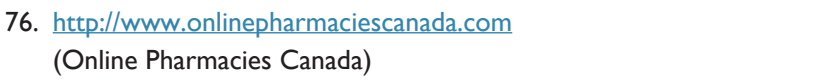 & Original site active & Original site active & Original site active \\
\hline 77. http://www.paylessmeds.com (PayLessMeds.com) & Original site active & $\begin{array}{l}\text { Redirect to } \\
\text { CanadaDrugs.com }\end{array}$ & $\begin{array}{l}\text { Redirect to } \\
\text { CanadaDrugs.com }\end{array}$ \\
\hline 78. http://www.peoplerx.com (PeopleRx.com) & Original site active & Original site active & Original site active \\
\hline
\end{tabular}


Table I (Continued)

\begin{tabular}{|c|c|c|c|}
\hline Website (name) & February 2006 & July 2009 & July 2010 \\
\hline $\begin{array}{l}\text { 79. http://www.pharmacycanada.biz } \\
\text { (Pharmacy Canada; MedCenter Canada) }\end{array}$ & Original site active & $\begin{array}{l}\text { Redirect to } \\
\text { CanadaDrugs.com }\end{array}$ & $\begin{array}{l}\text { Redirect to } \\
\text { CanadaDrugs.com }\end{array}$ \\
\hline 80. http://www.pharmacy-mailorder.com (International Meds) & Original site active & Original site active & Original site active \\
\hline 81. http://www.pharmacy-online.ca (PharmacyOnline) & Original site active & Original site active & Original site active \\
\hline 82. http://www.pharmacyrx.com (PharmacyRx.com) & Original site active & URL not found & URL not found \\
\hline $\begin{array}{l}\text { 83. http://www.pharmacyrxworld.com } \\
\text { (www.pharmacyRxworld.com) }\end{array}$ & Original site active & Original site active & Original site active \\
\hline 84. http://www.polarmeds.com (PolarMeds.com) & Original site active & Original site active & Original site active \\
\hline 85. http://www.prescriptionpoint.com (PrescriptionPoint) & Original site active & Original site active & Original site active \\
\hline 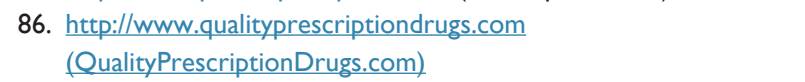 & Original site active & Original site active & Original site active \\
\hline 87. http://www.redwooddrugs.ca (Redwood Drugs) & Original site active & URL not found & URL not found \\
\hline 88. http://www.rfdrugstore.com (RF drugstore.com) & Original site active & Original site active & Original site active \\
\hline 89. http://www.rxl.biz (RxI.biz Pharmacy Inc) & Original site active & $\begin{array}{l}\text { Redirect to } \\
\text { CanadaDrugs.com }\end{array}$ & $\begin{array}{l}\text { Redirect to } \\
\text { CanadaDrugs.com }\end{array}$ \\
\hline 90. http://www.rxaccess.net (RxAccess) & Original site active & URL not found & URL not found \\
\hline 91. http://www.rxcanadapharmacy.com (RxCanadaPharmacy) & Original site active & $\begin{array}{l}\text { Not active; redirect } \\
\text { to domain directory }\end{array}$ & $\begin{array}{l}\text { Not active; redirect } \\
\text { to domain directory }\end{array}$ \\
\hline 92. http://www.rxcanadiansavings.com (MedCenterPlus+) & Original site active & URL not found & URL not found \\
\hline 93. http://www.rxcarecanada.com (RxCareCanada.com) & Original site active & $\begin{array}{l}\text { Redirect to } \\
\text { CanadaDrugs.com }\end{array}$ & $\begin{array}{l}\text { Redirect to } \\
\text { CanadaDrugs.com }\end{array}$ \\
\hline 94. http://www.rxdiscount.net (RxDiscount.net) & Original site active & Original site active & Original site active \\
\hline 95. http://www.rxdiscountcanada.com (Rx Discount Canada) & Original site active & URL not found & URL not found \\
\hline 96. http://www.rxnorth.com (RxNorth.com) & Original site active & $\begin{array}{l}\text { Redirect to } \\
\text { CanadaDrugs.com }\end{array}$ & $\begin{array}{l}\text { Redirect to } \\
\text { CanadaDrugs.com }\end{array}$ \\
\hline 97. http://www.rxtrustpharmacy.com (Rx Trust Pharmacy Canada)* & Original site active & Original site active & Original site active \\
\hline 98. http://www.sanjoychatterjee.com (Canada Drugs Online) & Original site active & $\begin{array}{l}\text { Not active; redirect } \\
\text { to domain directory }\end{array}$ & $\begin{array}{l}\text { Not active; redirect } \\
\text { to domain directory }\end{array}$ \\
\hline 99. http://www.secure-carerxsavings.com (Secure Care) & Original site active & Original site active & Original site active \\
\hline 100. http://www.securepharmacysite.com (CanadaDrugMart.com) & Original site active & URL not found & URL not found \\
\hline I0I. http://www.seniormedications.com (SeniorMedications.com)* & Original site active & Original site active & $\begin{array}{l}\text { Redirect to } \\
\text { Saverxpharmacy.com }\end{array}$ \\
\hline 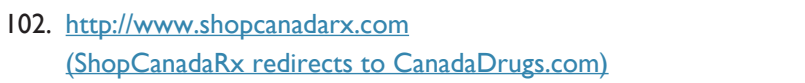 & Original site active & $\begin{array}{l}\text { Redirect to } \\
\text { CanadaDrugs.com }\end{array}$ & $\begin{array}{l}\text { Redirect to } \\
\text { CanadaDrugs.com }\end{array}$ \\
\hline 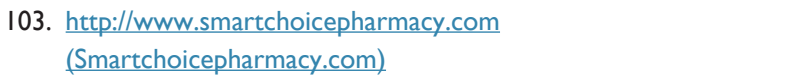 & Original site active & Original site active & Original site active \\
\hline 104. http://www.smartmed.ca (Smart Med Canada) & Original site active & $\begin{array}{l}\text { Redirect to } \\
\text { CanadaDrugs.com }\end{array}$ & $\begin{array}{l}\text { Redirect to } \\
\text { CanadaDrugs.com }\end{array}$ \\
\hline 105. http://www.squaremeds.com (Squaremeds.com)* & Original site active & Original site active & Original site active \\
\hline 106. http://www.texmeds.com (Texmeds.com) & Original site active & URL not found & URL not found \\
\hline $\begin{array}{l}\text { 107. http://www.thecanadianpharmacy.com } \\
\text { (The-Canadian-Pharmacy) }\end{array}$ & Original site active & Original site active & Original site active \\
\hline 108. http://www.truenorthpharmacy.com (TrueNorthPharmacy.com) & Original site active & URL not found & URL not found \\
\hline 109. http://www.universaldrugstore.com (Universal Drugstore) & Original site active & Original site active & Original site active \\
\hline I I0. http://www.usadrugonline.com (USADrugOnline.com) & Original site active & URL not found & URL not found \\
\hline III. *http://www.wmpharmacy.com (Wyandotte Medical Pharmacy) & Original site active & Original site active & Original site active \\
\hline $\begin{array}{l}\text { II2. http://www.worldcanadianpharmacy.com } \\
\text { (World Canadian Pharmacy.com) }\end{array}$ & Original site active & Original site active & Original site active \\
\hline $\begin{array}{l}\text { I13. https://ssl.perfora.net/lifhaus.canadianadrugs.com } \\
\text { (Lifhaus Medicines) }\end{array}$ & Original site active & URL not found & URL not found \\
\hline I14. https://www.allcanadianmeds.com (AllCanadianMeds.com) & Original site active & $\begin{array}{l}\text { Not active; redirect } \\
\text { to domain directory }\end{array}$ & $\begin{array}{l}\text { Not active; redirect } \\
\text { to domain directory }\end{array}$ \\
\hline II5. https://www.canadadiscountrx.com (Canada Discount Rx) & Original site active & $\begin{array}{l}\text { Redirect to } \\
\text { CanadaDrugs.com }\end{array}$ & $\begin{array}{l}\text { Redirect to } \\
\text { CanadaDrugs.com }\end{array}$ \\
\hline I 16. https://www.planetdrugsdirect.com (Planetdrugsdirect.com) & Original site active & Original site active & Original site active \\
\hline II7. https://www.thriftymeds.com (ThriftyMedsNow.com) & Original site active & Original site active & Original site active \\
\hline
\end{tabular}

Note: $\mathrm{An} *$ denotes that the company advertises pharmaceutical products from international markets in addition to Canadian products. Abbreviation: URL, uniform resource locator. 
and 5 months from the original active sites), one additional website was found to be a "dead" site and an additional four sites were found to be redirected sites, or redirected the visitor to another online Canadian pharmacy site. The information is presented in Table 2 .

After analysis of the remaining active 59 purportedly Canadian websites, almost 7\% advertise medications originating from countries other than Canada, in violation of both US and Canadian law. ${ }^{11}$ It is difficult to determine with certainty which sites sell pharmaceuticals made in countries other than Canada until an actual purchase is made and the product is delivered.

\section{Discussion}

Approximately 4 years and 5 months after initial posting, approximately half of the websites maintained information from the original state. Approximately $20 \%$ of the sites were forwarded to another Canadian Internet pharmacy site. Almost 20\% could not be found or had moved with no forwarding URL. It appears that links are terminated as websites are moved or removed, servers close down, or there are changes in business operations. In some instances, Internet pharmacy operations are perhaps shut down by legal authorities (Figure 1).

A tactic employed by several prominent Canadian Internet pharmacies is the automatic redirect to another Canadian Internet pharmacy site, which occurred in a significant number $(20 \%)$ of cases, at time periods after the original active sites were identified. This strategy is not uncommon in the Internet world. For some search engines, it can take weeks or months for even an authentic website to become available in the search engine listings. However, if an astute website developer can purchase the domain name of a similar yet now out-of-business company, consumers visiting the website for the nonfunctioning company will be delivered to a site that offers the same products and/or services, and both the consumer and the company will find what they are looking for.
One company in particular that employs this tactic is CanadaDrugs.com. ${ }^{12}$ CanadaDrugs.com is a legitimate Internet pharmacy operation licensed in Canada by the Manitoba Pharmaceutical Association. ${ }^{13}$ Similar to several business operations in this report, because it operates outside the jurisdiction of the US Food and Drug Administration, it is considered an illegitimate operation in the US. ${ }^{14}$

Based on their advertising taglines, CanadaDrugs.com claims to be "Canada's largest" online pharmacy. ${ }^{12}$ In fact, that claim could be, at least in part, justified by this study. Of the 24 websites encountered during this study that automatically redirect to another site, 14 of those websites (58.3\%) redirect to $\underline{\text { CanadaDrugs.com. }}$. Of the websites that redirect to CanadaDrugs.com, none of the sites notify the consumer that they are being redirected to another website operated by another company.

Although difficult to pinpoint the precise number of Canadian Internet pharmacies in operation, estimates have ranged to as high as 120 to 140 sites. ${ }^{15}$ A number of sites, some of which were identified in this study, claim to be "Canadian Internet pharmacies" but are actually advertising and selling pharmaceuticals made in foreign countries and that are illegal to sell in Canada. This tactic has been coined "hiding under the maple leaf" and refers to websites that advertise themselves as Canadian or prominently display the familiar maple leaf flag but are actually registered elsewhere. ${ }^{16}$

Drug importation from Canada via the Internet has received much attention as a remedy for consumers for the high price of prescription drugs. ${ }^{17}$ Sales of drugs from Canadian pharmacies to US customers are estimated at more than $\$ 1$ billion per year. ${ }^{17}$ US consumers who shop at Canadian Internet pharmacies to purchase their prescription medications reportedly include senior citizens, the uninsured or under insured, and those who must pay "out of pocket."10

According to the National Association of Boards of Pharmacy (NABP), 97\% of the over 10,000 Internet drug

Table 2 Condition of Canadian Internet pharmacy websites at three different time periods

\begin{tabular}{llll}
\hline Condition of website & Number of sites (\%) & & \\
\cline { 2 - 4 } & February 2006 & July 2009 & July 20 I0 \\
\hline Original active Internet pharmacy website & $117(100 \%)$ & $64(54.7 \%)$ & $59(50.4 \%)$ \\
Redirect to other Canadian Internet pharmacy site & $0(0 \%)$ & $20(17.4 \%)$ & $24(20.5 \%)$ \\
Redirect to domain name directory site & $0(0 \%)$ & $12(10.3 \%)$ & \\
(not an Internet pharmacy site) & & & \\
"Dead" website (original site not found) & $0(0 \%)$ & $21(17.9 \%)$ & \\
Total nonfunctioning websites & $0(0 \%)$ & $33(28.2 \%)$ & \\
Totals & $117(100 \%)$ & $117(100 \%)$ & $34(29.1 \%)$ \\
\hline
\end{tabular}




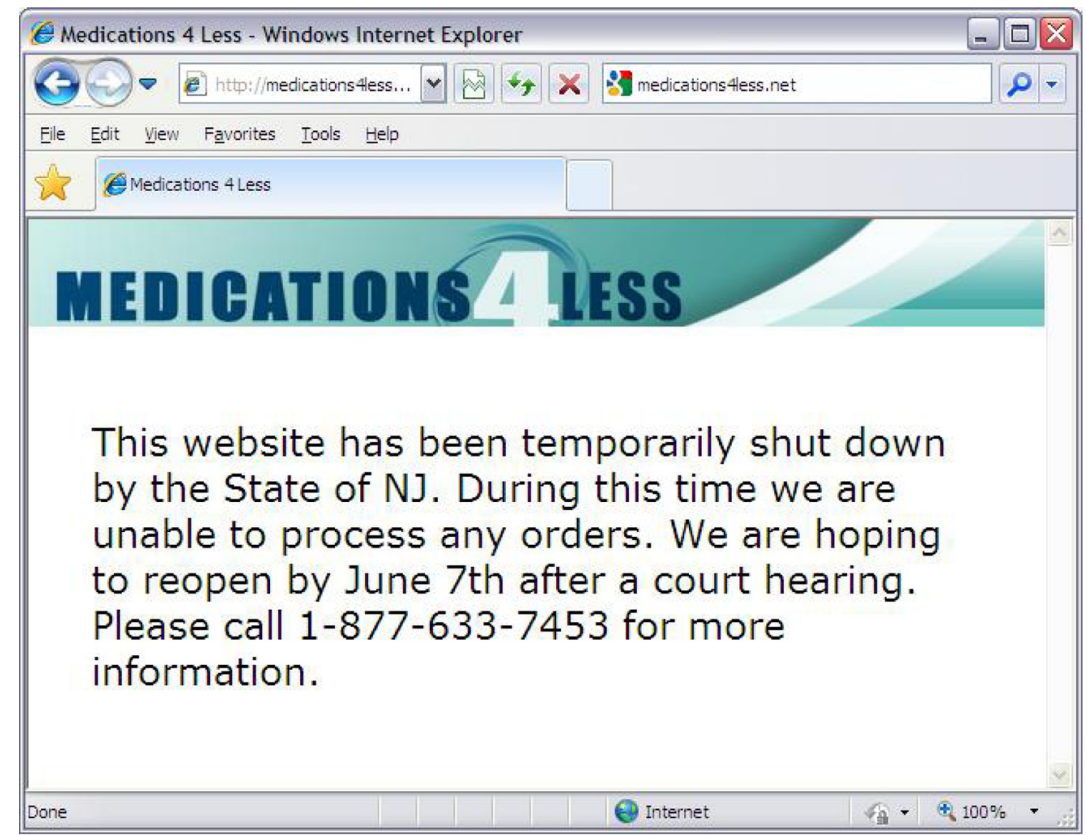

Figure I A message on an Internet pharmacy website indicating unavailability due to legal action.

Notes: A note presumably left by the company indicating that the website had been "shut down by the state of NJ (New Jersey)" and would possibly reopen after a June 7 court date. Since the site remained inaccessible, it can be surmised that the results of the legal proceeding were not favorable.

Table 3 Selected comments by consumers regarding unavailability of Internet pharmacies

"What happened to Pharmacy Escrow?? Website says they are closed?"

"Ugh, tell me about it! I went there today to describe the ordering process in my blog..only to find their message. I can't believe it! Luckily, I found about www.alldaychemist.com looks reputable, so that's who l'll try next."

"I received an e-mail from In House Pharmacy that they have been shut down by LegitScript.com's program, which is trying to ban ALL online pharmacies outside the US. They have opened under "Inhousepharmacy.biz". Are people as individuals going to be targeted as well for purchases from these online pharmacies outside the US?"

"Ordered prescription online. Received call the next day, trouble with credit card, asked for Western Union payment. Call the next day, they are out of product I ordered. They offered to sell larger bottle for $\$ 220$ more. Refused to substitute for a similar product. Refused a refund. Hung up on me multiple times. I am out $\$ 300$ with nothing to show for it. Now their web site is under construction. They have opened new site at www.lifecaremeds.com with phone number 805-500-434I. These guys are real crooks."

"Officially close - www.4rx.com

And now I see 365 pills.com is also gone ...

Bad start 2012."

"And another big dinosaur of the biz is going down.

Well these are bad days for the industry.

It was, is and stays: An individual have only the right to be alive and healthy as long it have money.

Online pharmacies which are selling cheap and appropiate generics have to be hunted down, due they are helping the poor and nearly worthless inidviduals to live and spread themself. Thats the reality.

To be an affiliate or operator of an online pharmacy it is not the profit which makes you work,

it is the abandonment to make the world better!"

"Inhousepharmacy.com has been a major and important resource to the trans community for many years. While that url has been shut down, they have found a new one, inhousedrugstore.biz, and are currently operating under it, offering an opening special of $10 \%$ off all prescriptions for the month of October.

So long as the vast majority of doctors are utterly ignorant of trans healthcare needs AND require we pay them large sums of money to sign off on something we already know, gray market options with a proven history, like inhouse, are very important. I do see this as a wakeup call that resources like this wont always be around if we don't advocate for them - or for more permanent solutions to accessible and affordable healthcare."

\section{Source}

http://hcgdietinfo.com/hcgdietforums/

f8/pharmacy-escrow-closed-41435/

http://hcgdietinfo.com/hcgdietforums/

f8/pharmacy-escrow-closed-4I435/

http://www.skincaretalk.com/t/l7162/ oh-no-in-house-pharmacy-shut-down

http://www.ripoffreport.com/ pharmacies/24online-storecom/24online-store-comlifecaremed-746II.htm

http://rxaffiliateforum.com/ showthread.php?738I-Did-4rx.comclose-down http://rxaffiliateforum.com/ showthread.php?738I-Did-4rx.comclose-down

http://www.bilerico.com/2010/09/ inhousepharmacycom_stops shipping to the us.php 
outlets assessed may be illegitimate or illegal, and out of compliance with pharmacy laws and practice standards. ${ }^{18}$ It may actually be beneficial for consumers if illegal pharmacy websites disappear or their domains are ceased so that they may be referred to legitimate Internet pharmacy operations. But it appears that the desire to purchase drugs, coupled with the power to do so, legally or illegally, is not subsiding. With chronic illness on a steep increase, drug therapy is likely to remain a mainstay of treatment and the demand for drugs even greater in the future. ${ }^{19}$

In general, when a patient receives health care services on a regular basis, and when those services are disrupted and/ or discontinued, it would not be unreasonable to assume that there may be detrimental effects to the patient's wellbeing (Table 3). One consideration for patients if Internet pharmacy sites were suddenly inaccessible would be the potential disruption to continuity of care.

Continuity of care is concerned with the quality of care over time. ${ }^{20}$ It suggests cooperative involvement between patient and physician, but in a broader sense can mean "ongoing health care management toward the goal of high quality, cost-effective medical care." ${ }^{21}$

Hypothetically, for a 3 month supply of drug (offered by most Internet pharmacies), a patient would need to visit a site at least four times a year. In our 4 year and 5 month time period, a patient would have visited a site 17 to 18 times to obtain prescription drugs on a regular basis. For a returning customer, a consequence of website attrition may be disruption of care.

\section{Conclusion}

Inaccessible websites are not uncommon, so when encountered, a general recommendation for "link rot" is to search for the information resource, rather than the URL address that points to the missing site. ${ }^{6}$ Based on the results of this study, this suggestion may not successfully be applied to loss of Canadian Internet pharmacy websites.

\section{Acknowledgments}

The authors wish to thank the Texas Affiliate of the American Heart Association for their financial support in the preparation of this research report. Special thanks to Ngoc Phung, Pharm D, for assistance with the preparation of this manuscript.

\section{Disclosure}

The authors declare no conflict of interest in this work.

\section{References}

1. Broken links: just how rapidly do science education hyperlinks go extinct? [webpage on the Internet]. Markwell J [update July 24, 2006]. Available from: http://www-class.unl.edu/biochem/url/broken_links. html. Accessed December 5, 2012.

2. Aronsky D, Madani S, Carnevale RJ, Duda S, Feyder MT. The prevalence and inaccessibility of Internet references in the biomedical literature at the time of publication. $J$ Am Med Inform Assoc. 2007;14(2):232-234.

3. Carnevale RJ, Aronsky D. The life and death of URLs in five biomedical informatics journals. Int J Med Inform. 2007;76(4):269-273.

4. Dellavalle RP, Hester EJ, Heilig LF, et al. Information science. Going, going, gone: lost Internet references. Science. 2003;302(5646): 787-788.

5. Rumsey M. Runaway train: problems of permanence, accessibility, and stability in the use of web sources in law review citations. Law Library J. 2002;94:27-35.

6. Veronin MA. Where are they now? A case study of health-related Web site attrition. J Med Internet Res. 2002;4(2):E10.

7. Markwell J, Brooks DW. "Link rot" limits the usefulness of web-based educational materials in biochemistry and molecular biology. Biochem Mol Biol Educ. 2003;31(1):69-72.

8. Hunter T. E-Pharmacy: A Guide to the Internet CareZone. American Pharmaceutical Association. Washington, DC: 2002:191-225.

9. Orizio G, Merla A, Schulz PJ, Gelatti U. Quality of online pharmacies and websites selling prescription drugs: a systematic review. $J$ Med Internet Res. 2011;13(3): 74 .

10. Veronin MA. Canadian Internet pharmacies: price, policy, and perspective. Res Social Admin Pharm. 2007;3:236-248.

11. US Food and Drug Administration. FDA operation reveals many drugs promoted as "Canadian" products really originate from other countries [press release]. FDA News; 2005 [December 16]. Available from: http:// www.fda.gov/NewsEvents/Newsroom/PressAnnouncements/2005/ ucm108534.htm. Accessed April 24, 2013.

12. CanadaDrugs.com [homepage on the Internet]. Available from: http:// www.canadadrugs.com. Accessed December 5, 2012.

13. Pharmacychecker.com [homepage on the Internet]. Available from: http://www.pharmacychecker.com. Accessed April 22, 2013.

14. Inspections, compliance, enforcement, and criminal investigations [webpage on the Internet]. US Food and Drug Administration [updated September 20, 2012]. Available from: http://www.fda.gov/ICECI/ EnforcementActions/WarningLetters/2012/ucm321068.htm. Accessed April 22, 2013.

15. Kaldy J. Drug importation: the call of the north. Caring for the ages. A monthly newspaper for long-term care practitioners. July 2004;3-4:7.

16. Petrecca L. No prescription? No Problem. Prevention.com. Available from: http://news.heartland.org/sites/all/modules/custom/heartland_ migration/files/pdfs/14390.pdf. Accessed April 22, 2013.

17. Morgan S, Hurley J. Internet pharmacy: prices on the up-and-up. CMAJ. 2004;170(6):945-946.

18. National Association of Boards of Pharmacy (NABP). Available from: http://www.nabp.net/programs/accreditation/vipps/faqs\#5. Accessed November 26, 2012.

19. Hagstrom B, Mattsson B, Wimo A, Gunnarsson RK. More illness and less disease? A 20-year perspective on chronic disease and medication. Scand J Public Health. 2006;34(6):584-588.

20. Gulliford M, Naithani S, Morgan M. What is 'continuity of care'? $J$ Health Serv Res Policy. 2006;(11)4:248-250.

21. Continuity of care, definition of [webpage on the Internet]. American Academy of Family Physicians. Available from: http:/www.aafp.org/ online/en/home/policy/policies/c/continuityofcaredefinition.html. Accessed April 22, 2013. 
Drug, Healthcare and Patient Safety

Dovepress

\section{Publish your work in this journal}

Drug, Healthcare and Patient Safety is an international, peer-reviewed open-access journal exploring patient safety issues in the healthcare continuum from diagnostic and screening interventions through to treatment, drug therapy and surgery. The journal is characterized by the rapid reporting of reviews, original research, clinical, epidemiological and

post-marketing surveillance studies, risk management, health literacy and educational programs across all areas of healthcare delivery. The manuscript management system is completely online and includes a very quick and fair peer-review system. Visit http://www.dovepress.com/ testimonials.php to read real quotes from published authors.

Submit your manuscript here: http://www.dovepress.com/drug-healthcare-and-patient-safety-journal 